OPEN ACCESS

Edited by:

Hans Dierckx,

Ghent University, Belgium

Reviewed by:

Winfried Mayr,

Medical University of Vienna, Austria

Jieyun Bai,

The University of Auckland, New

Zealand

${ }^{*}$ Correspondence:

Ki Moo Lim

kmlim@kumoh.ac.kr

Specialty section: This article was submitted to

Biomedical Physics,

a section of the journal

Frontiers in Physics

Received: 15 December 2017 Accepted: 04 December 2018

Published: 18 December 2018

Citation:

Heikhmakhtiar AK, Rasyidin FA and Lim KM (2018) V241F KCNQ1

Mutation Shortens Electrical

Wavelength and Reduces Ventricular Pumping Capabilities: A Simulation

Study With an Electro-Mechanical Model. Front. Phys. 6:147.

doi: 10.3389/fphy.2018.00147

\section{V241F KCNQ1 Mutation Shortens Electrical Wavelength and Reduces Ventricular Pumping Capabilities: A Simulation Study With an Electro-Mechanical Model}

\author{
Aulia Khamas Heikhmakhtiar ${ }^{1}$, Fakhmi Adi Rasyidin ${ }^{2}$ and Ki Moo Lim ${ }^{1 *}$ \\ ${ }^{1}$ Computational Medicine Lab, Department of IT Convergence Engineering, Kumoh National Institute of Technology, Gumi, \\ South Korea, ${ }^{2}$ Computational Medicine Lab, Department of Industrial Engineering, Kumoh National Institute of Technology, \\ Gumi, South Korea
}

Death due to ventricular fibrillation (VF) can occur over a relatively short time period. During the first stage, an irregular heartbeat or arrhythmia of the heart may occur. Therefore, studying arrhythmia could reveal important insights relevant to the prevention of VF. One of the factors known to cause arrhythmia is the generation of mutations in the ion channels of myocytes. The current experimental methods to monitor and observe subjects with arrhythmia are invasive, and could possibly harm the subject with no guarantee of obtaining good results. These limitations could be overcome by using an extensively validated computational simulation study. This study aims to enhance our understanding of the effect of the $\mathrm{V} 241 \mathrm{~F}$ mutation on electromechanical behavior in the heart. We simulated three conditions; wild-type (WT), heterozygous/intermediate V241F, and pure V241F conditions in an electrophysiological single cell model and three-dimensional electro-mechanics ventricular model. The electro-mechanics model is a one-way coupling of the electrical compartment to the mechanical compartment by $\mathrm{Ca}^{2+}$ transient concentration. Consistent with a previous study, the $\mathrm{V} 241 \mathrm{~F}$ mutation significantly shortened the action potential duration at 90\% repolarization (APDgo) under pure V241F mutation conditions, due to the gain of function of the slow delayed rectifier potassium $\left(I_{K_{S}}\right)$ channel. This APDgo shortening is associated with a short electrical wavelength, which shortens the $\mathrm{Ca}^{2+}$ activation time as well. The hemodynamic responses showed that the V241F mutation lowered ventricular contraction under normal sinus rhythm conditions by decreasing the stroke volume, stroke work, and ejection fraction. During reentry, the $\mathrm{V} 241 \mathrm{~F}$ mutation significantly reduced the ventricular contractility compared with the WT condition. In conclusions, the effect of the two variants of V241F (intermediate and pure) mutation not only disturbed the electrophysiological events but also affected the mechanical behavior significantly. The result of this study can be used as a reference for the cardiovascular expert to decide the appropriate pharmacology of $I_{K S}$ conductance block for the patient.

Keywords: V241F KCNQ1 mutation, electromechanical model, sinus rhythm, ventricular fibrillation, computational model 


\section{INTRODUCTION}

Recent data from the American Heart Association showed that cardiovascular diseases are the primary causes of death worldwide [1]. Sudden cardiac death and ventricular fibrillation (VF) are associated with a $<11 \%$ chance of survival. VF occurs when the heart quivers instead of performing a normal contraction. This results in a reduction of blood distribution within the circulatory system [2]. VF causes death within a short period $(<1 \mathrm{~h})$ in the absence of any observable symptoms [3]. In the last two decades, the number of deaths from VF worldwide has reached 300,000 per year [3-5]. Since VF is initiated by ventricular arrhythmia [5-7], the study of arrhythmia is of great of importance. Over the last few decades, researchers have found that disturbance in the electrical activity of the heart is due to ion channel mutations, also known as ion channelopathies [8, 9].

Ion channels are protein complexes that act as gates in the cardiomyocytes membrane and sarcolemma, regulating the permeability of specific ions through the membrane [10]. The ion channels in myocytes dynamically control the ionic currents that orchestrate action potentials (APs). Changes in the electrical properties of myocytes directly affect their mechanical activation [11]. Hence, changes in the properties of ion channels due to mutations will presumably change the AP shape, as well as alter the myocytes. In addition, mechanical activation of the myocytes will also affect the electrophysiological events related to stretch-activated channels (mechano-electric feedback) [12]. Understanding the mechanism of electrical disturbance in the early stages of VF could help clinicians make decisions regarding the optimal therapies.

In 2014, a novel mutation in the slow delayed rectifier potassium $\left(\mathrm{K}^{+}\right)$channel $\left(I_{K s}\right)$ was found. The mutation p.Val241phe (V241F) occurs in the S4 transmembrane domain of the KCNQ1 gene in Korean families with a history of atrial fibrillation and bradycardia [13]. $I_{K s}$ are composed of both KCNQ1 (KVLQT1) channel subunits and KCNE1 (minK) subunits in myocytes and are involved in the repolarization of the AP during the plateau phase and refractory period [14]. V241F is a gain of function mutation of KCNQ1 that abbreviates the action potential duration at $90 \%$ repolarization $\left(\mathrm{APD}_{90}\right)$. A short $\mathrm{APD}_{90}$ results in short QT syndrome and is one of the primary causes of VF [15]. Our previous study enhanced understanding of the electrophysiological activity of the V241F mutation by using an image-based three-dimensional (3D) cardiac model [16]. In this study, we observed the contribution of the V241F mutation to ventricular pumping of the heart by using a $3 \mathrm{D}$ electro-mechanic ventricular model.

The goal of this study was to computationally predict the electrical activity and mechanical responses of human ventricles with the V241F mutation under conditions of normal sinus rhythm and VF. The experimental study of arrhythmia has many limitations because it is difficult to measure the electrical and mechanical activity of the heart non-invasively; furthermore, only the surface can be observed. Hence, we used an extensively validated computational model as an alternative to uncover the effect of the V241F mutation on the electrical and mechanical activity of the heart. To the best of our knowledge, no study has observed the contribution of the $\mathrm{V} 241 \mathrm{~F}$ mutation to the electrophysiological activity of the heart and the mechanical responses of the ventricle. Here, we simulated an electrophysiological single cell model, and a 3D electromechanical model of a failing ventricle under wild-type (WT), intermediate V241F, and pure V241F mutation conditions.

\section{MATERIALS AND METHODS}

In this study, we used an electromechanical model of failing ventricle based on magnetic resonance imaging with finite element combined with the lumped-parameter model, similar to our previous study [17]. The methods for the electromechanical coupling we used in this study are based on those described by Gurev et al. [18, 19]. The present electromechanical model consists of electrical and mechanical properties that were weakly coupled by intracellular calcium $\left(\mathrm{Ca}^{2+}\right)$ transient. Physiologically, the electrical propagation in the ventricular tissue represents depolarization of each cell throughout the ventricle. The depolarization in the myocytes activates the $\mathrm{Ca}^{2+}$ channel of the sarcoplasmic reticulum, releasing $\mathrm{Ca}^{2+}$ into the cytosol. The $\mathrm{Ca}^{2+}$ then binds to troponin $\mathrm{C}$, causing myofilament sliding to occur. This event also called cross-bridge contraction, which generates active tension resulting in deformation of the ventricles. The intracellular $\mathrm{Ca}^{2+}$ transient acts as the trigger to convert the electrical activation into a mechanical phenomenon. In our simulation, we obtained the $\mathrm{Ca}^{2+}$ information from the electrophysiological model from each myocardial cell and input it into the contractile myofilament dynamics model.

\section{Electrical Model}

The electrical mesh consists of 241,725 nodes with $1,298,751$ elements forming in a tetrahedral shape. The mesh was embedded with Purkinje networks model, based on the study of Berenfeld and Jalife, on the endocardial boundary [20]. The Purkinje fiber was constructed from 2D line mesh, which then mapped onto the endocardial surface of the $3 \mathrm{D}$ ventricular mesh. The attachment of the Purkinje network was used only in the simulation under normal sinus rhythm condition. The electrical simulation includes multi-level electrophysiological activity: single cell activation and the propagation of the electrical signal in the ventricle tissue. The electrical propagation is expressed by solving a mono-domain partial differential equation which presented the current flow in the ventricles composed of myocytes connected by gap junctions. The single cell activation is expressed by solving a set of the ordinary differential equation which expressed the current flow through ion channels, pumps, and exchanger in the membrane and the sarcoplasmic reticulum. The electrophysiological cell model incorporated into our model was based on the work of ten Tusscher et al. [21] which includes epicardium, M-cell, and endocardium elements. The cell membrane is represented as a capacitor, and the ion channels are represented as the resistance. Ions traversing the cell membrane are represented as an electrical current passing through the capacitor and resistors. The electrophysiological behavior of this model can be described using the following differential equation 
from Hodgkin and Huxley [22]:

$$
C_{m} \frac{d V_{m}}{d t}=-\left(I_{i o n}+I_{s t i m}\right)
$$

Where $V$ is voltage, $t$ is time, $I_{i o n}$ is the sum of all transmembrane ionic currents, $I_{\text {stim }}$ is the external current stimulus, and $C_{m}$ is the cell capacitance per unit surface area. The total ionic current $I_{i o n}$ is represented by:

$$
\begin{aligned}
I_{i o n} & =I_{N a}+I_{K 1}+I_{t o}+I_{K r}+I_{K s}+I_{C a, L}+I_{N a, C a}+I_{N a, K}+I_{p, C a} \\
& +I_{p, K}+I_{b, C a}+I_{b, N a}
\end{aligned}
$$

Where $I_{N a}$ is the fast sodium current, $I_{K 1}$ is the inward rectifier potassium current, $I_{t o}$ is the transient outward potassium current, $I_{K r}$ and $I_{K s}$ are rapid delayed rectifier and slow delayed rectifier potassium current, respectively, $I_{C a L}$ is the L-type inward calcium current, $I_{\mathrm{NaCa}}$ is the sodium-calcium exchanger current, $I_{\mathrm{NaK}}$ is the sodium-potassium pump current, $I_{p C a}$ is the plateau calcium current, $I_{p K}$ is the plateau potassium current, $I_{b \mathrm{Ca}}$ is the background calcium current, and $I_{b N a}$ is the background sodium current. Based on the single cell model, the equation was modified to model the electrophysiological phenomena in $3 \mathrm{D}$ space. The $3 \mathrm{D}$ electrical propagation phenomenon can be described with the following partial differential equation:

$$
\frac{d V}{d t}=-\frac{I_{i o n}+I_{\text {stim }}}{C_{m}}+\frac{1}{\rho_{x} S C_{m}} \frac{\partial^{2} V}{\partial x^{2}}+\frac{1}{\rho_{y} S C_{m}} \frac{\partial^{2} V}{\partial y^{2}}+\frac{1}{\rho_{z} S C_{m}} \frac{\partial^{2} V}{\partial z^{2}}
$$

where $\rho$ is the cellular resistivity with respect to the $x, y$, and $z$ directions, and $S$ is surface to volume.

To implement the properties of the V241F mutation into the signal cell and three-dimensional electrophysiological model, we modified the $I_{K s}$ of the ten Tusscher cell model with the equation of V241F mutation. We implemented the V241F mutation equation by Ki et al. which has been validated with experimental data [13].

\section{Mechanical Model}

The contraction and deformation of the ventricles were simulated according to the mechanical properties. To initiate myocytes contraction, $\mathrm{Ca}^{+2}$ arising from the electrical simulation under the V241F mutation was used as the input. Contraction of the ventricle was the result of active tension, as represented by the myofilament dynamics model of Rice et al. [23]. Ventricular deformation is described by the stress equilibrium equations of passive cardiac mechanics, with the myocardium assumed to be an orthotropic, incompressible, and hyperelastic material that has passive properties defined by an exponential strain energy function [24]. The mechanical mesh consists of 356 nodes with 172 Hermite (non-linear) elements. The ventricular contraction was calculated by solving the active myofilament model equations simultaneously with passive cardiac mechanics equation on each node of the finiteelement mesh. The Rice et al. model is well-known to have a very good understanding describing excitation-contraction coupling phenomenon. The mechanical ventricular model was integrated with the lumped parameter model. The lumped model represented the systemic and pulmonic circulatory system based on a study by Kerckhoffs et al. [25]. The ATP consumption presented here is based on the rates of ATP consumed for myofilament contraction. The ATP consumption rate $(E)$ is the outcome of cross-bridge detachment rate $\left(g_{x b T}\right)$ and the single overlap fraction of thick filaments $\left(\right.$ SOVF Thick $_{\text {: }}$

$$
E=g_{x b T} \times S O V F_{T h i c k}
$$

\section{Simulation Protocol}

For the single cell simulation model, we applied a stimulus to the cell 30 times with a basic cycle length (BCL) of $1 \mathrm{~s}$. The strength of the stimulus was $52 \mathrm{pA} / \mathrm{pF}$ and it was applied for $0.2 \mathrm{~ms}$. After the cell reached a steady-state condition, we obtain the $\mathrm{APD}_{90}$, $I_{K s}$ current, and APD restitution curve toward BCL and diastolic interval (DI) under WT, intermediate V241F, and pure V241F mutation conditions. $\mathrm{APD}_{90}$ was defined as the interval between the depolarization and repolarization at $90 \%$ of the AP upstroke, measured from the top.

Using a 3D electromechanical model, we analyzed the electrical propagation wave under two conditions: normal sinus rhythm and reentry/arrhythmia. We decreased the CV of the electrical propagation to $60 \mathrm{~cm} / \mathrm{s}$, and in the finite element model, we multiplied the constant of passive scaling level by 5 in the strain energy function to increase the stiffness of the myocardium tissue. During normal sinus pacing, the electrical stimulation was applied using a Purkinje network model with BCL of $600 \mathrm{~ms}$ for all three cases. The electrical signal propagation comes from the Purkinje networks is expressed by solving one-dimensional wave propagation equation which triggered the ventricle tissue activation. The simulation lasted for $42 \mathrm{~s}$ in order to reach a steady state was reached.

To simulate the reentry condition in $3 \mathrm{D}$ ventricular model, we used the standard S1-S2 protocol. S1 is an electrical stimulus which applied three times at the apex with $600 \mathrm{~ms}$ BCL. The S1 stimulus generated three planar wave-forms toward the base of the ventricle. The S2 is the protocol which reset the membrane potential in half of the ventricular medium to resting state. The S2 was applied just before the head of the third planar wave reach the base area. This protocol will allow the reentry to be generated toward the resting potential state nodes. The simulation time for the reentry condition was 10 s under the WT, intermediate V241F, and pure V241F mutation conditions.

Next, we coupled the transient $\mathrm{Ca}^{2+}$ data from the electrical simulation with those of the mechanical simulation. The mechanical simulation results for the normal sinus rhythm condition was taken from the last cycle of the simulation/steadystate. For the reentry, we used all $10 \mathrm{~s}$ data to compare the mechanical responses among the WT, intermediate V241F, and pure V214F mutation conditions during reentry. See the Supplementary Material regarding the electro-mechanical coupling technics. 

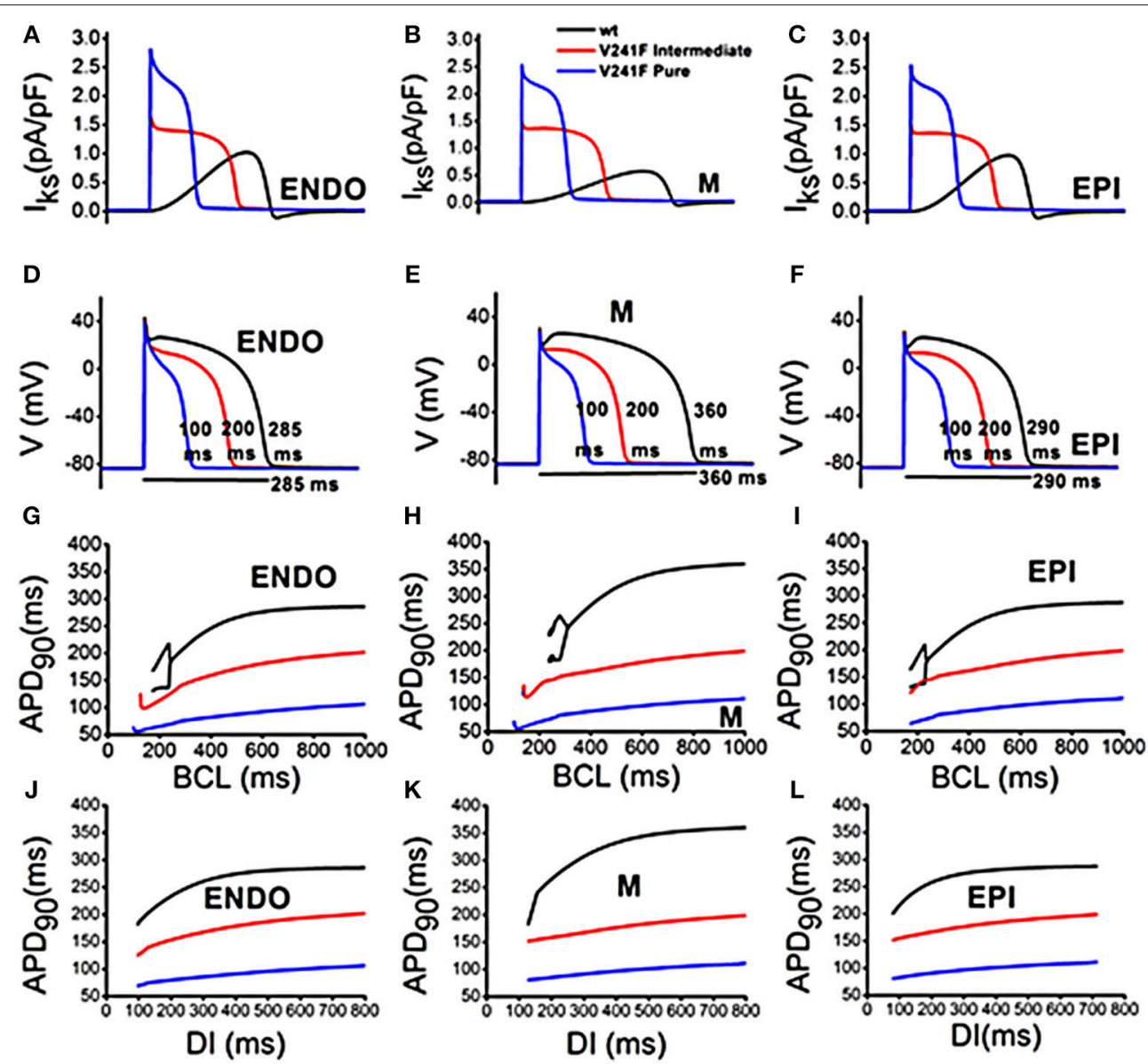

FIGURE 1 | Cardiac single cell response of WT and variants of intermediate and pure V241F mutation condition in 3 type of cell: (A) Slow delayed rectifier $\mathrm{K}^{+}$current $\left(I_{K_{S}}\right)$ in endocardial cell (B) Slow delayed rectifier $K^{+}$current $\left(I_{K_{S}}\right)$ in mid-myocardial cell (C) Slow delayed rectifier $K^{+}$current $\left(I_{K S}\right)$ in epicardial cell (D) APD profile in endocardial cell (E) APD profile in mid-myocardial cell (F) APD profile in epicardial cell (G) APD according to BCL in endocardial cell (H) APD according to BCL in mid-myocardial cell (I) APD according to BCL in epicardial cell (J) APD according to DI in endocardial cell (K) APD according to DI in mid myocardial cell (L) APD according to DI in epicardial cell.

Under the reentry condition, the spiral wave will be induced to the ventricular tissue. The altered activation of the electrical signal desynchronized the contractility of the ventricle. Hence the chaotic condition was exhibited, and quivering of the heart altered the LV pressure, aortic pressure, blood volume in the ventricles, and ATP consumption rates.

\section{RESULTS}

\section{Single Cell Responses}

Figure 1 compares cellular responses among the WT, V241F intermediate V241F, and pure V241F mutation conditions including $I_{K s}$ (Figures 1A-C), AP shape (Figures 1D,E), APD toward BCL (Figures 1D,E), and APD toward Di (Figures 1J-L). Figures 1A-C shows that V241F pure mutation has the highest $I_{K s}$ density compared to intermediate and WT conditions. Notice that the $I_{K s}$ current was activated during the depolarization for both intermediate and pure V241F conditions. This early activation of the $I_{K s}$ is consistent with the findings of Ki et al. in the atrium cell endowed with the V241F mutation [13]. Normally the $I_{K s}$ activated during repolarization. The pure V241F current was deactivated earlier compared to that under the intermediate and WT conditions. Figures $1 \mathrm{D}-\mathbf{F}$ shows that the $\mathrm{APD}_{90}$ of the pure mutation was 100 and $200 \mathrm{~ms}$ under the intermediate conditions in endo, $\mathrm{M}$-cell, and epicedial cell, respectively. In the ten Tusscher model, they differentiate the conductance of $I_{K s}$ and $I_{t o}$ to generate the AP shape for endo, $\mathrm{M}$, and epicardium cell. Under the intermediate and pure V241F conditions, the $I_{K s}$ was activated in the earlier phase of AP, leaving its role at the repolarization time. As the result, the shape of AP for endo, $\mathrm{M}$, and epicardium under intermediate and pure V241F become uniform. This means that the $I_{K s}$ has more significant impact than the $I_{t o}$ to compose the endo, M, and epi AP shape. In the comparison of APD toward the BCL, the alternant was observed in the M-cell under intermediate and V241F pure mutation at the 150 and $175 \mathrm{~ms}$ BCL, respectively.

Figure 2 shows the $\mathrm{CV}$ restitution curves under WT, intermediate and pure V241F mutation conditions. At BCL 700, 


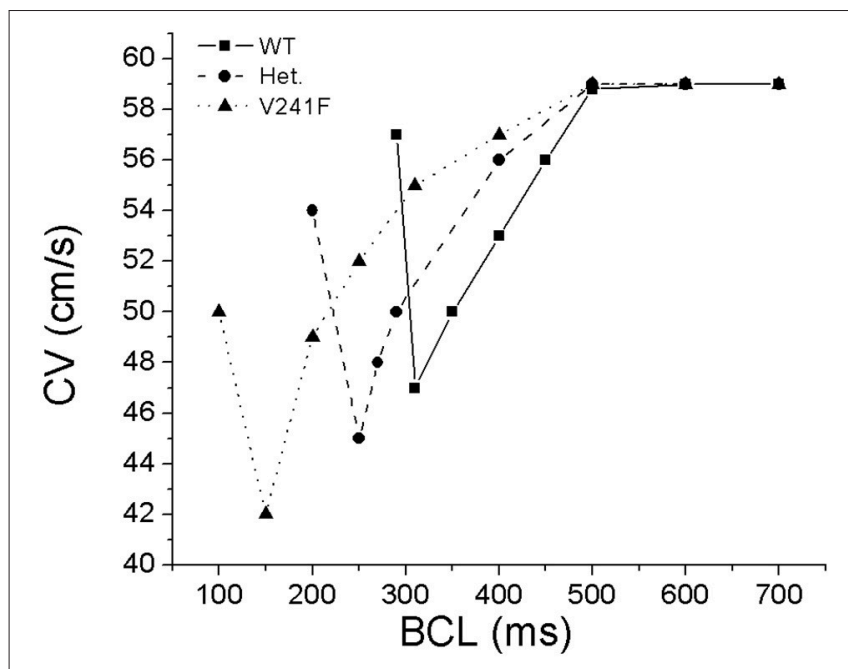

FIGURE 2 | Conduction velocity restitution curves obtained from a cable shape mesh by pacing at the left side of the mesh for 100 time under WT, intermediate (notified by Het.), and pure V241F mutation conditions.

600 , and 500, the CV was all the same for the three conditions, $59 \mathrm{~cm} / \mathrm{s}$. The $\mathrm{CV}$ of the pure V241F mutation was decreased to $42 \mathrm{~cm} / \mathrm{s}$ until BCL $150 \mathrm{~ms}$. The CV of the pure V241F mutation was unstable at BCL $100 \mathrm{~ms}$. The $\mathrm{CV}$ of the intermediate was remained to decrease until BCL $250 \mathrm{~ms}$. However, the $\mathrm{CV}$ of the intermediate was increased at BCL 200. The CV of the WT was decreased steadily until BCL $300 \mathrm{~ms}$. The CV was increased at BCL $290 \mathrm{~ms}$. The increased BCL at the last point was due to alternant during the stimulation. The cell failed to generate action potential at the even stimuli. In results, the AP generation was skipped one cycle and exhibiting double BCL from what it is supposed to.

\section{Three-Dimensional Ventricular Responses During Normal Sinus Rhythm}

Figure 3 represents the transmembrane potential propagation in the $3 \mathrm{D}$ ventricular model during one cycle $(600 \mathrm{~ms})$ of sinus pacing. Even though all stimulations started at the same time, the pure V241F mutation condition showed a faster repolarization phase compared with the intermediate V241F and WT conditions. After the Purkinje firing the electrical signal to the ventricular tissue, under the $\mathrm{V} 241 \mathrm{~F}$ pure mutation condition, the membrane potential already in the repolarization phase at $140 \mathrm{~ms}$ indicated by the yellow color. Under the intermediate condition, the wavelength completely gone at the $340 \mathrm{~ms}$. Under the WT condition, the wavelength was sustained to more than $340 \mathrm{~ms}$.

Figure 4 shows the electrical activation time (EAT) that was put into our electromechanical model of ventricular heart failure (Figure 4A) and electrical deactivation time (EDT) (Figure 4B) for WT, V241F intermediate and pure mutation during one cycle of sinus rhythm. The EAT was the same under all three conditions (see Figure 4A). However, the EDT under the WT condition was longer compared with that of the intermediate V241F and pure V241F mutation conditions (Figure 4B). The pure V241F mutation condition had the lowest EDT which is associated with the short wavelength. The EDT results indicated that the pure V241F mutation had faster repolarization than the intermediate V241F and WT conditions. The maximum and minimum EAT and EDT values for all three conditions are given in Figure 4C.

Table 1 shows the ventricular mechanical responses during normal sinus pacing (one cycle) under the WT, intermediate $\mathrm{V} 241 \mathrm{~F}$, and pure V241F mutation conditions. The stroke volume (SV), stroke work (SW), and ejection fraction (EF) were decreased under intermediate $\mathrm{V} 241 \mathrm{~F}$ mutation condition and further decreased under the pure V241F mutation condition. The SV reach $44 \mathrm{~mL}$ under the WT condition, and 36 and $32 \mathrm{~mL}$ under the intermediate $\mathrm{V} 241 \mathrm{~F}$ and the pure $\mathrm{V} 241 \mathrm{~F}$ mutation conditions, respectively. SW indicates the performance of ventricle during contraction. The SW of WT condition was $4,566 \mathrm{mmHg} \mathrm{mL}$. In the case of pure V241F mutation, the SW indicated the lowest performance, having a value of 3,315 $\mathrm{mmHg} \mathrm{mL}$. The contractile adenosine three phosphate (ATP) consumption rates under the intermediate V241F mutation and WT conditions were 92 and $166 \mathrm{~s}^{-1}$, respectively. Under the pure mutation condition, the ATP consumption rate dropped to $84 \mathrm{~s}^{-1}$.

\section{Three-Dimensional Ventricular Responses During Reentry}

Figure 5A shows the snapshot view of arrhythmia under the WT, intermediate V241F, and pure V241F mutation conditions. In our simulation, the reentry wave was sustained until the end of simulation for all three condition. Nevertheless, the center of rotation of the spiral wave appeared in a different place for each conditions. However, from the snapshot, we can see that pure V241F mutation condition had the shortest wavelength among all three conditions. This accorded with the $\mathrm{APD}_{90}$ of the pure V241F mutation also being the shortest $\mathrm{APD}_{90}$ (Figure 5B).

Figure 6 illustrates the mechanical responses, including the LV pressures (Figure 6A), the LV volume (Figure 6B), LV pressure-volume relation (Figure 6C), and contractile ATP consumption rates (Figure 6D) during $10 \mathrm{~s}$ of reentry under the WT, intermediate V241F, and pure V241F mutation conditions. The figures on the right-hand side correspond to the last $1 \mathrm{~s}$ of the mechanical responses, i.e., the steady state condition. According to the LV pressure data, the peak ventricular pressure was $30 \mathrm{mmHg}$ for the pure V241F mutation, $35 \mathrm{mmHg}$ for the intermediate V241F condition, and $42 \mathrm{mmHg}$ for the WT condition. The short $\mathrm{APD}_{90}$ under the mutation conditions resulted in reduced calcium activation in the myocytes; thus, the mutation conditions showed poorer contractility. A short wavelength under the mutation conditions caused faster reentry and increased the electrical activation rate, thus producing a more chaotic condition. We can see from the LV pressure graph that the pure V241F mutation condition had the highest ventricular beating frequency over $1 \mathrm{~s}$ (right side of Figure 6). The ventricular beat rate was $420 \mathrm{bpm}$ for the pure V241F mutation, $300 \mathrm{bpm}$ for the intermediate V241F mutation, and $240 \mathrm{bpm}$ for the WT condition. 
A

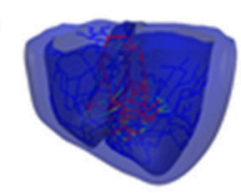

B

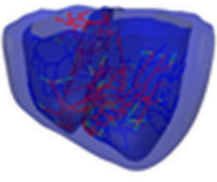

C

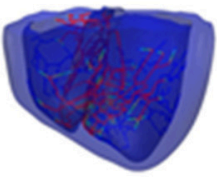

$40 \mathrm{~ms}$
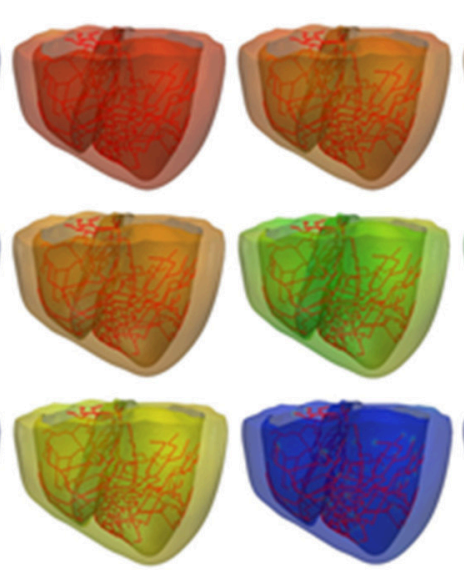

$240 \mathrm{~ms}$

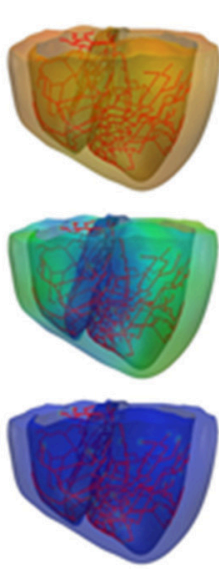

$270 \mathrm{~ms}$

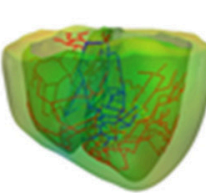
Vollage $30(\mathrm{Vm})$
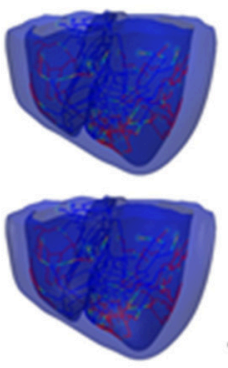

$340 \mathrm{~ms}$

FIGURE 3 | Snapshots of transmural distribution of membrane potential during one cycle of sinus pacing. (A) WT, (B) V241F Intermediate, (C) V241F Pure conditions in the 3D ventricular tissue model.
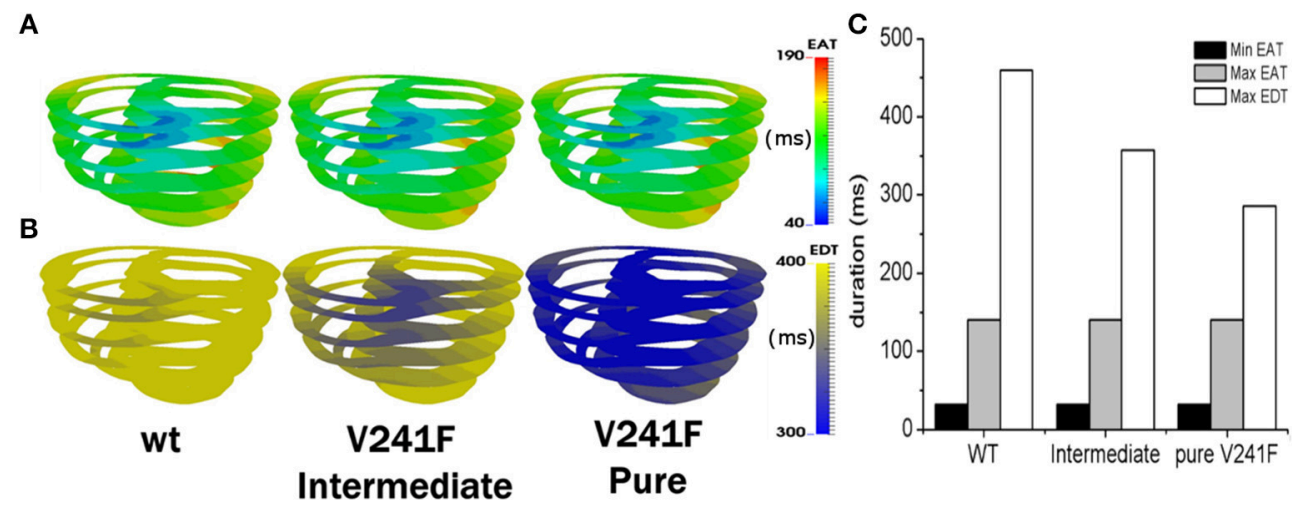

FIGURE 4 | EAT during one cycle of sinus pacing for WT, V241F intermediate and Pure Condition. (A) EAT mapped on ventricle model, (B) EDT mapped on ventricle model. (C) Comparison graph of minimum EAT, maximum EAT, and maximum EDT.

According to LV volume graph (Figure 6B), the pure V241F mutation had the highest blood volume in the LV. The pure V241F mutation had a blood volume of $55 \mathrm{~mL}$ when it reached the steady-state of reentry. However, the intermediate V241F and WT conditions had similar steady state blood volumes of 34 and $33 \mathrm{~mL}$, respectively. The larger volume in the pure V241F mutation condition was related to the LV pressure (Figure 6A); under this condition, the ventricle had the lowest contractility, which was insufficient for ejecting the blood.

Figure 6C shows the LV pressure-volume relation under reentry condition for all three cases. As the figure shows, the pure V241F mutation condition had the highest volume, while its LV pressure was the lowest compared to the intermediate V241F mutation and WT conditions, representing a very weak contraction. Thus, under a steady state of reentry condition (Figure 6C right side), it shows that the pure mutation has the smallest amount of SV and SW. The LV pressure under the intermediate V241F mutation condition ranged at $24-35 \mathrm{mmHg}$ with LV volume ranged at $32-34 \mathrm{~mL}$. Under the WT condition, the LV pressure ranged at $21-42 \mathrm{mmHg}$ with the LV volume ranged at $31-33 \mathrm{mmHg}$.

Figure 6D shows the ATP consumption rate for all three cases during reentry. The contractile ATP consumption rate for the pure V241F mutation was the lowest, which accord with the PV curves results (see Figure 6C) showing that, because of pure mutation condition relatively little energy could be consumed, the pressure of the ventricle was insufficient to pump the blood efficiently.

During reentry or fibrillation, because of the chaotic electrical events, the hemodynamics of the heart was seriously altered under the WT, intermediate, and pure V241F mutation conditions (Figure 6). All three conditions showed poor pumping contraction capabilities with the pure V241F being the worst condition. The frequency of the LV pressure was very high with the blood remaining steady in the LV. 


\section{DISCUSSION}

Using a single cell and three-dimensional electromechanical model of human ventricle, this study investigated the effect of the V241F KCNQ1 mutation during normal sinus rhythm and reentry using a computational method. The method to couple the electrical and the mechanical compartment as well as the lumped circulatory model is based on the study of Gurev et al. [18, 19]. This is the first study to implement a finite element-based electromechanical model for the heart to investigate cardiac electrophysiology and the mechanism underlying the V241F KCNQ1 mutation. The major findings of our research are as follows:

1. Similarly to the atrial cell by Ki et al. [13], in a single cell simulation that was applied to three types of ventricle cellular model (endo, M-cell, epi), V241F mutation activates the $I_{K s}$ channel during depolarization phase (activated early) and it

TABLE 1 | Ventricular mechanical responses during sinus rhythm under WT, V241F intermediate, and V241F pure mutation conditions.

\begin{tabular}{lccc}
\hline Variant type & WT & V241F intermediate & V241F pure \\
\hline End diastolic volume $(\mathrm{mL})$ & 76 & 83 & 85 \\
End systolic volume $(\mathrm{mL})$ & 32 & 47 & 53 \\
Stroke volume $(\mathrm{mL})$ & 44 & 36 & 32 \\
LV systolic pressure $(\mathrm{mmHg})$ & 125 & 127 & 127 \\
Stroke work (mmHg $\mathrm{mL})$ & 4,566 & 3,693 & 3,315 \\
ATP consumption rate $\left(\mathrm{s}^{-1}\right)$ & 163 & 92 & 84 \\
Mean left atrial pressure $(\mathrm{mmHg})$ & 14 & 13 & 15 \\
Mean aortic pressure $(\mathrm{mmHg})$ & 99 & 99 & 102 \\
Ejection fraction $(\%)$ & 58 & 43 & 38 \\
\hline
\end{tabular}

increased the $I_{K s}$ density (Figures 1A-C). This is the caused for the short $\mathrm{APD}_{90}$.

2. Under the 3D model of normal sinus rhythm condition, repolarization showed faster acceleration during sinus pacing under the V241F pure mutation condition compared with the intermediate V241F mutation and WT conditions (Figure 3). With the same CV, the EAT of the three cases were the same except for the EDT (Figure 4). The SV, SW, and EF under sinus rhythm were lowered under the intermediate condition, even worst under that V241F pure mutation condition (Table 1).

3. During the reentry phase, the pure V241F mutation generated the shortest membrane potential propagation wave (Figure 5A) among all three conditions.

4. The mechanical response of pure V241F mutation has the lowest LV pressure, highest LV volume, and lowest ATP consumption rates during reentry.

The single cell electrophysiological responses showed that the KCNQ1 mutation activates the $I_{K s}$ channel during depolarization time with a high density of current. This early activation force the vast amount of positive ions to leave early out of the myocytes abbreviated the APD during repolarization time significantly (Figure 1). These results are consistent with the study by Ki et al. which of the mutation was occurred in the atrium cell [13]. The $\mathrm{APD}_{90}$ shortening due to the V214F mutation makes the short wavelength hence short QT wave. This increases the fatal effect since the short QT syndrome is associated with VF which could lead to sudden cardiac death [15]. The wavelength shortening owing to the mutation was observed under normal sinus rhythm and reentry conditions (Figures 3, 5).

The low performance of ventricular contractility under sinus rhythm condition is described in Table 1. The pure V241F mutation showed the lowest SV, SW, EF, and ATP consumption

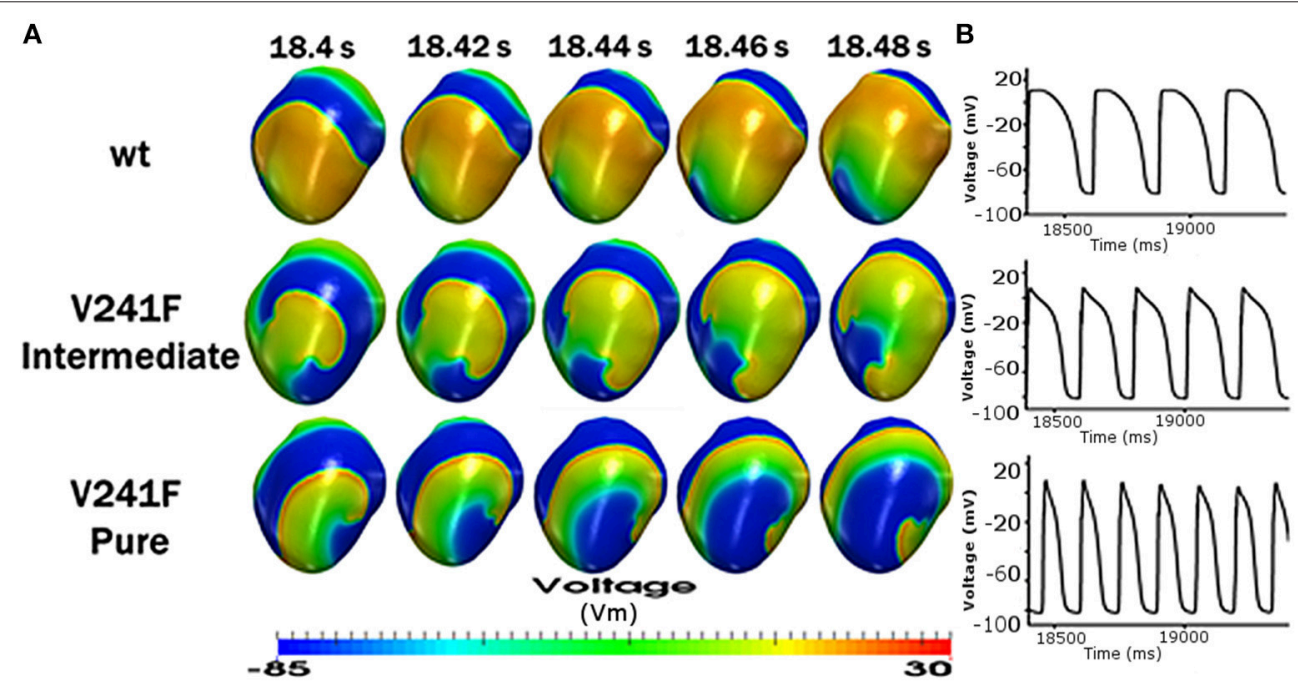

FIGURE 5 | Reentrant dynamics wave in the 3D ventricular tissue model for WT and V241F mutation conditions. (A) Snapshot of trans mural distribution of wt condition (top), Snapshot of trans mural distribution of V241F Intermediate (center), Snapshot of trans mural distribution of V241F Pure (bottom) of membrane potential; and (B) time traces of action potentials (AP) in $1 \mathrm{~s}$. 

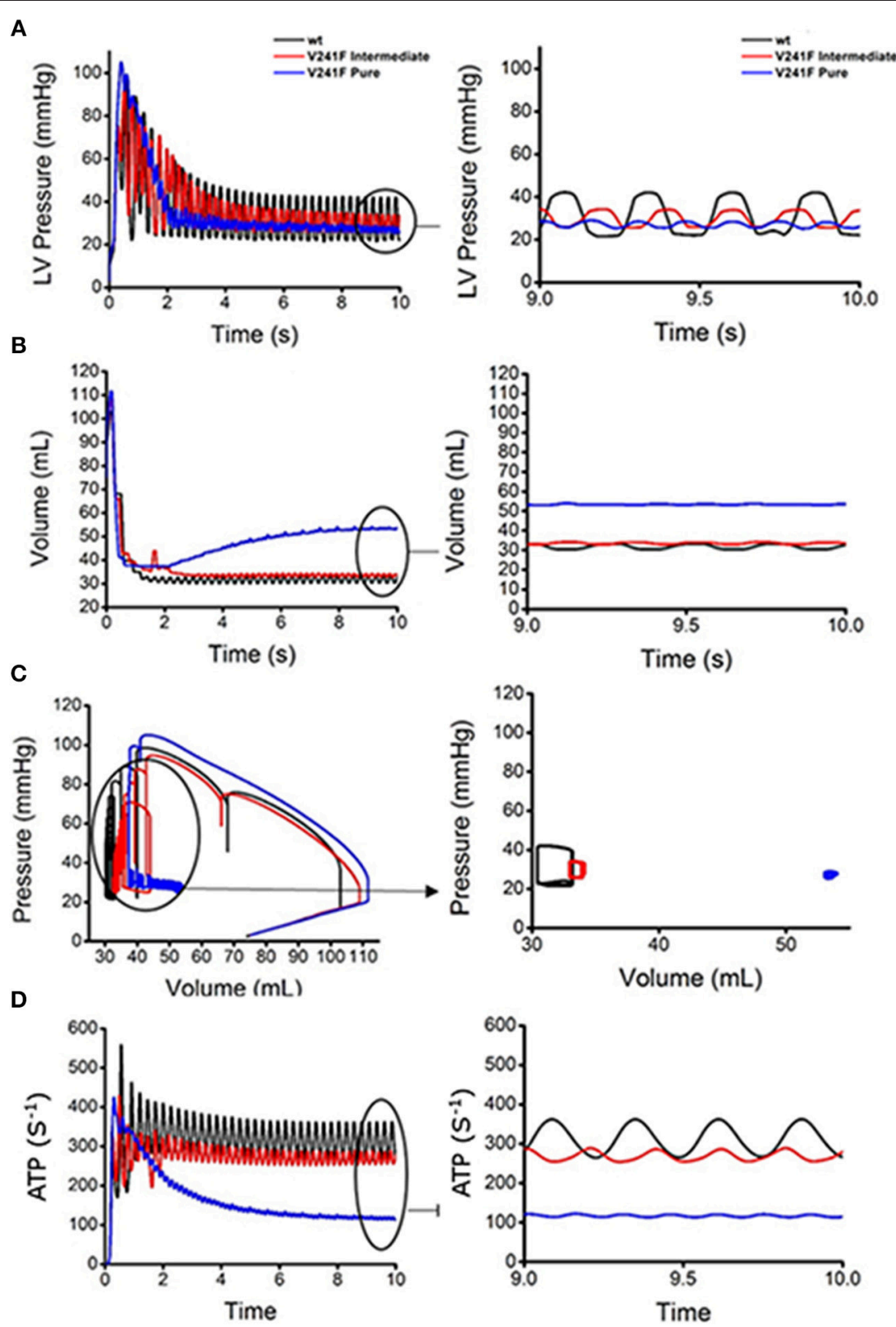

FIGURE 6 | Ventricular mechanical responses of reentrant dynamics wave in the 3D ventricular tissue model for WT, V241F intermediate and pure condition. (A) LV pressure waveforms, (B) LV volume waveforms, (C) LV pressure-volume curves and (D) ATP curves for WT, V241F Intermediate, and Pure mutation conditions.

rates compared to the intermediate V241F mutation and WT conditions. The poor activation of the mechanical responses is related to the APD abbreviation. Short APD means the time for $\mathrm{Ca}^{+2}$ activation was also decreased. Since the $\mathrm{Ca}^{+2}$ is the trigger for myofilament sliding or cross-bridge activation, short $\mathrm{Ca}^{+2}$ activation time would decrease the ventricular pumping efficacy.

During reentry, the $\mathrm{V} 241 \mathrm{~F}$ mutations (intermediate and pure) shortened the electrical propagation wavelength (Figure 5A) and increased the frequency of the electrical activation especially to the nodes close to the center of the rotor (Figure 5B). Under the WT condition, the wavelength was two times longer to that under pure mutation condition. The AP shape and the frequency of the activation at specific node were aligned in Figure 5.

To compare the hemodynamic responses under the WT, intermediate, and pure V241F mutation during reentry, we present the LV pressure waveform, LV volume waveform, LV Pressure-Volume (PV) curve, and contractile ATP consumption rate. Our results showed that the V241F mutation (intermediate and pure) decreased the LV pressure (Figure 6A) and ATP consumption rate (Figure 6D) during reentry. Because the pressure of the LV was low, there was insufficient strength to pump the blood out of the ventricles, resulting in the 
accumulation of blood inside the ventricles (Figure 6B). The relationship between the pressure and volume waveforms of the $\mathrm{LV}$ is described by the PV curve. The pure V241F mutation condition showed the smallest loop during reentry (Figure 6C). The pressure's waveforms vary with the ATP consumption rate among all three conditions (Figure 6D).

Our results show that the electrophysiological activation is strongly related to the ventricular pumping under both sinus rhythm contraction and reentry scenarios under WT, intermediate, and V241F pure mutation conditions. The alteration to the electrical activation would cause abnormality of the hemodynamics of the heart. In this case, the shortening of $\mathrm{APD}_{90}$ due to the $\mathrm{V} 241 \mathrm{~F}$ mutation lowered the pumping performance of that under the intermediate condition and even more with that under pure mutation condition.

This study demonstrated mechanistically the effect of the V241F mutation on the electrical activation and also mechanical pumping behavior under sinus rhythm and reentry condition. The gain of function of the $I_{K s}$ channel lowers the pumping efficacy by altering the $\mathrm{APD}_{90}$, thus shortened the electrical activation wavelength. The clinical impact of this study is that the ratio of the $\mathrm{APD}_{90}$ shortening in the myocytes by the $\mathrm{V} 241 \mathrm{~F}$ mutation can be used as a reference for the cardiologist expert to decide the appropriate pharmacology of $I_{K s}$ conductance block for the patient. Previous studies indexed pharmacological substances related to voltage-gated potassium channels [26, 27]. Although further investigation, such as in vivo or in vitro test should be conducted.

\section{LIMITATIONS}

There were several limitations to this study that should be addressed. We used isotropic conductivity for the electrical propagation through tissue. The electromechanical model we used is a one-directional coupling model. We coupled the electrical activation to the mechanical contraction by giving the mechanical model the information of the $\mathrm{Ca}^{2+}$ transient from the electrical simulation. The mechano-electrical feedback was not considered in this study. For example, stretch-activated channel [28] was not implemented and the information of contractile energy consumption was not fed back to the electrical model. The energy consumption was calculated to quantify the mechanical activity of the ventricle. The experimental study about the V241F

\section{REFERENCES}

1. Benjamin EJ, Blaha MJ, Chiuve SE, Cushman M, Das SR, Deo R, et al. Heart disease and stroke statistics-2017 update: a report from the American Heart Association. Circulation (2017) 135:e146-603. doi: 10.1161/CIR.0000000000000485

2. Jalife J. Ventricular fibrillation: mechanisms of initiation and maintenance. Ann Rev Physiol. (2000) 62:25-50. doi: 10.1146/annurev.physiol.62.1.25

3. Zipes DP, Wellens HJ. Sudden cardiac death. Circulation (1998) 98:2334-51. doi: 10.1161/01.CIR.98.21.2334

4. Myerburg R, Kessler K, Interian A Jr., Fernandez P, Kimura S, Kozlovskis P, et al. Clinical and experimental pathophysiology of sudden cardiac death. In: Cardiac Electrophysiology: From Cell to Bedside, 1st Edn. Philadelphia, PA: WB Saunders Co (1990). p. 666-78. mutation on the ventricle, to our best knowledge, is not available yet. Hence it becomes a constraint to explicitly validate this computational prediction.

In conclusions, the effect of the two variants of V241F (intermediate and pure) mutation not only disturbed the electrophysiological events but also affected the mechanical behavior significantly. Within a single cell, the pure V241F mutation shortened the ventricular $\mathrm{APD}_{90}$ of $\mathrm{M}$-cell by 73 and $45 \%$ under the pure and intermediate V241F mutations, respectively. In the $3 \mathrm{D}$ electrical model of normal sinus rhythm and reentry, the APD of the intermediate and pure V241F mutation conditions was significantly shortened vs. the WT, which prone to VF situation. As a result, the mechanical responses associated with a normal sinus rhythm under the V241F mutation conditions consumed less ATP, and showed a decreased EF, SV, and SW. Hemodynamic function during reentry was impaired by the $\mathrm{V} 241 \mathrm{~F}$ mutation, resulting in very weak heart contractility and an accumulation of blood in the LV.

\section{AUTHOR CONTRIBUTIONS}

$\mathrm{AH}$ ran the simulation, organized the results, and constructed and wrote the manuscript according to the theory and the results of the study. FR ran parts of the simulation of this study. KL guided and supervised the authors starting from the theoretical study, simulation, and finalization of the paper.

\section{ACKNOWLEDGMENTS}

This research was partially supported by the MSIT (Ministry of Science, ICT), under the ITRC (Information Technology Research Center) support program (IITP-2018-2014-000639) supervised by the IITP, and NRF (National Research Foundation) under basic engineering research project (2016R1D1A1B0101440) and the EDISON (NRF-2011-0020576) Programs.

\section{SUPPLEMENTARY MATERIAL}

The Supplementary Material for this article can be found online at: https://www.frontiersin.org/articles/10.3389/fphy. 2018.00147/full\#supplementary-material

5. Weiss JN, Garfinkel A, Karagueuzian HS, Qu Z, Chen P-S. Chaos and the transition to ventricular fibrillation. Circulation (1999) 99:2819-26. doi: 10.1161/01.CIR.99.21.2819

6. Chen PS, Garfinkel A, Weiss JN, Karagueuzian HS. Spirals, chaos, and new mechanisms of wave propagation. Pacing Clin Electrophysiol. (1997) 20:41421. doi: 10.1111/j.1540-8159.1997.tb06200.x

7. Karma A. Electrical alternans and spiral wave breakup in cardiac tissue. Chaos (1994) 4:461-72. doi: 10.1063/1.166024

8. Hsiao P-Y, Tien H-C, Lo C-P, Juang J-MJ, Wang Y-H, Sung RJ. Gene mutations in cardiac arrhythmias: a review of recent evidence in ion channelopathies. Appl Clin Genet. (2013) 6:1-13. doi: 10.2147/TACG. S29676

9. Wilde AA, Bezzina CR. Genetics of cardiac arrhythmias. Heart (2005) 91:1352-8. doi: 10.1136/hrt.2004.046334 
10. Roden DM, Balser JR, George AL Jr, Anderson ME. Cardiac ion channels. Ann Rev Physiol. (2002) 64:431-75. doi: 10.1146/annurev.physiol.64.083101.145105

11. Shih H-T. Anatomy of the action potential in the heart. Tex Heart Inst J. (1994) 21:30-41.

12. $\mathrm{Hu} \mathrm{H}$, Sachs F. Stretch-activated ion channels in the heart. J Mol Cell Cardiol. (1997) 29:1511-23. doi: 10.1006/jmcc.1997.0392

13. Ki C-S, Jung CL, Kim H-j, Baek K-H, Park SJ, On YK, et al. A KCNQ1 mutation causes age-dependant bradycardia and persistent atrial fibrillation. Pflügers Arch. (2014) 466:529-40. doi: 10.1007/s00424-0131337-6

14. Sanguinetti M, Curran M, Zou A, Shen J, Specter P, Atkinson D, et al. Coassembly of KvLQT1 and $\operatorname{minK}$ (IsK) proteins to form cardiac IKs potassium channel. Nature (1996) 384:80-3. doi: 10.1038/384 080a0

15. Gussak I, Brugada P, Brugada J, Wright RS, Kopecky SL, Chaitman BR, et al. Idiopathic short QT interval: a new clinical syndrome? Cardiology (2000) 94:99-102. doi: 10.1159/000047299

16. Imaniastuti R, Lee HS, Kim N, Youm JB, Shim EB, Lim KM. Computational prediction of proarrhythmogenic effect of the V241F KCNQ1 mutation in human atrium. Prog Biophys Mol Biol. (2014) 116:70-5. doi: 10.1016/j.pbiomolbio.2014.09.001

17. Lim KM, Constantino J, Gurev V, Zhu R, Shim EB, Trayanova NA. Comparison of the effects of continuous and pulsatile left ventricular-assist devices on ventricular unloading using a cardiac electromechanics model. $J$ Physiol Sci. (2012) 62:11-9. doi: 10.1007/s12576-011-0180-9

18. Gurev V, Constantino J, Rice J, Trayanova N. Distribution of electromechanical delay in the heart: insights from a threedimensional electromechanical model. Biophys J. (2010) 99:745-54. doi: 10.1016/j.bpj.2010.05.028

19. Gurev V, Lee T, Constantino J, Arevalo H, Trayanova NA. Models of cardiac electromechanics based on individual hearts imaging data. Biomech Model Mechanobiol. (2011) 10:295-306. doi: 10.1007/s10237-0100235-5

20. Berenfeld O, Jalife J. Purkinje-muscle reentry as a mechanism of polymorphic ventricular arrhythmias in a 3-dimensional model of the ventricles. Circ Res. (1998) 82:1063-77. doi: 10.1161/01.RES.82.10.1063
21. Ten Tusscher KH, Panfilov AV. Alternans and spiral breakup in a human ventricular tissue model. Am J Physiol Heart Circ Physiol. (2006) 291:H1088100. doi: 10.1152/ajpheart.00109.2006

22. Hodgkin AL, Huxley AF. A quantitative description of membrane current and its application to conduction and excitation in nerve. J Physiol. (1952) 117:500-44. doi: 10.1113/jphysiol.1952.sp004764

23. Rice JJ, Wang F, Bers DM, De Tombe PP. Approximate model of cooperative activation and crossbridge cycling in cardiac muscle using ordinary differential equations. Biophys J. (2008) 95:2368-90. doi: 10.1529/biophysj.107.119487

24. Holzapfel GA, Ogden RW. Constitutive modelling of passive myocardium: a structurally based framework for material characterization. Philos Trans R Soc Lond A (2009) 367:3445-75. doi: 10.1098/rsta.2009.0091

25. Kerckhoffs RC, Neal ML, Gu Q, Bassingthwaighte JB, Omens JH, McCulloch AD. Coupling of a 3D finite element model of cardiac ventricular mechanics to lumped systems models of the systemic and pulmonic circulation. Ann Biomed Eng. (2007) 35:1-18. doi: 10.1007/s10439-006-9212-7

26. Li G-R, Dong M-Q. Pharmacology of cardiac potassium channels. $A d v$ Pharmacol. (2010) 59. 93-134. doi: 10.1016/S1054-3589(10)59004-5

27. Tamargo J, Caballero R, Gómez R, Valenzuela C, Delpón E. Pharmacology of cardiac potassium channels. Cardiovasc Res. (2004) 62:9-33. doi: 10.1016/j.cardiores.2003.12.026

28. Hu Y, Gurev V, Constantino J, Bayer JD, Trayanova NA. Effects of mechanoelectric feedback on scroll wave stability in human ventricular fibrillation. PLoS ONE (2013) 8:e60287. doi: 10.1371/journal.pone.0060287

Conflict of Interest Statement: The authors declare that the research was conducted in the absence of any commercial or financial relationships that could be construed as a potential conflict of interest.

Copyright (c) 2018 Heikhmakhtiar, Rasyidin and Lim. This is an open-access article distributed under the terms of the Creative Commons Attribution License (CC BY). The use, distribution or reproduction in other forums is permitted, provided the original author(s) and the copyright owner(s) are credited and that the original publication in this journal is cited, in accordance with accepted academic practice. No use, distribution or reproduction is permitted which does not comply with these terms. 